\title{
Dengue Virus: A Review on Epidemiology, Clinical Manifestation, Diagnosis and Pathogenesis
}

\author{
Sourav Kumar Kundu ${ }^{1}$, Md Ferdous Seraj ${ }^{2}$, Md Mohasin ${ }^{1}$ and Tania \\ Rahman ${ }^{1 *}$ \\ ${ }^{1}$ Department of Biochemistry and Molecular Biology, University of Dhaka, Dhaka, Bangladesh \\ ${ }^{2}$ Department of Environmental Science and Management, North South University, Dhaka, \\ Bangladesh
}

${ }^{*}$ Corresponding author: Dr. Tania Rahman, Associate Professor, Department of Biochemistry and Molecular Biology, University of Dhaka, Dhaka, Bangladesh, Email: tania.rahman@du.ac.bd

\section{Review Article}

Volume 4 Issue 2

Received Date: July 15, 2020

Published Date: September 24, 2020

DOI: $10.23880 /$ jidtm- 16000142

\section{Abstract}

Dengue, a mosquito borne viral infection is a leading cause of acute flu-like illness occasionally develops into a potentially lethal complication. DENV, the virus responsible for causing dengue has become prevalent in recent years with a million of infection as well as a couple of thousand deaths in the tropical and subtropical climates worldwide. The four strains of dengue virus identified so far among which DENV-II and DENV-III are more prevalent in Bangladesh have some common distinctive symptoms. As dengue symptoms are most of the time not intense and sometimes asymptomatic, differentiating dengue patients from patients with acute febrile illness is a great challenge and it is tough to identify at the preliminary stage. The dramatic rise of global incidence of dengue infection has generated an urgent yet an unmet need for an accurate and validated rapid diagnostic test for DENV infection. In this review, we survey and analysis our current knowledge and relative understanding of the epidemiology of dengue virus, its classification, clinical manifestation of dengue fever, its pathogenesis and clinical diagnosis of dengue.

Keywords: Dengue virus; DENV infection; Flu; Flavivirus

\section{Introduction}

Dengue is recognized as one of the most fatal viral diseases with the potential to cause life-threatening infections in human. It is an arthropod-borne disease responsible for causing an array of clinical disease manifestations in humans. The Aedes aegypti mosquito is recognized as the most common vector of dengue [1]. In some regions of Asia, Aedes albopictus is found to act as a secondary vector to some extent. A single mosquito is able to transmit the virus, if it is infected and incubated for 4-10 days. The Aedes aegypti mosquito is normally found in urban areas and surprisingly only $10 \mathrm{~mL}$ of water is enough for this type of mosquito to breed. Unlike other mosquitoes, Aedes aegypti bites during daytime and before twilight. Dengue fever is undoubtedly the most frequent arboviral infection throughout the world. The majority of the world's population (2.5-3 billion people) lives in the regions which are marked as endemic for dengue. It is predicted that 50-80 million people get infected by dengue each year and 500,000 cases are of dengue hemorrhagic fever (DHF). It is unfortunate that at least 12,000-24,000 people die each year, most of which are children aged $<15$ years. Dengue has turned out to be one of the leading causes of child death in many Asian and South American countries [2]. The disease is prevalent in hot, steamy, sweltering tropical climates worldwide especially in semi-urban and urban regions.

In 1943, scientists Ren Kimura and Susumu Hotta first discovered the dengue virus. These two scientists studied 
blood samples of suspects taken during the 1943 dengue epidemic in Nagasaki, Japan. After a year, Albert B. Sabin and Walter Schlesinger individually isolated dengue virus. The virus isolated by both the scientists is now referred to as dengue virus serotype 1 (DEN-1).

\section{Epidemiology of Dengue}

Dengue is assumed to have been recorded first in a medical encyclopedia of the Chinese from the Dynasty called Chin Dynasty (265-420 AD). The Chinese surmised that the dengue was "water poison" and believed that its' spreading was somehow related with flying insects [3]. It transpired from Africa at the time of the slave trade from 15th to 19th centuries [4]. As this period was the age of exploration and rise of international trade, this disease got the chance to spread globally. During this period, dengue viruses were likely to be endemic in many urban and tropical areas. Epidemics that appeared like dengue and had similar disease pattern and dispersion occurred in 1635 in the West Indies and in 1699 in Central America [5].

\section{Dengue in Southeast Asia}

After the World War II, Dengue appeared as a burden in public health of Southeast Asia leading to major environmental disruption and demographic alteration [6]. As people shifted in large numbers with their equipment, Aedes aegypti got the opportunity to spread in new geographical regions. Egyptian, Japanese and Allied troops moving around the area have helped providing sensitive carriers for dengue virus. As the WWII propagated the geographic expansion of the Dengue virus along with its vector, it was further taken ahead by the urbanization during the post WWII period [7].

Due to disproportionate accommodation, poor sewerage system and inadequate water supply- which presented a perfect environment for highly domesticated Aedes aegypti, dengue appeared as one of the leading health issues in Southeast Asia. Currently, around $75 \%$ of globally dengue exposed people live in Asia pacific region [8]. Epidemiologically, Indian subcontinent has a very complex pattern of dengue fever with constantly varied, mutated prevalent strains, geographically distant regions and robustness over the last couple of decades. Dengue was first reported in India back in 1946 [9]. First dengue hemorrhagic fever outbreak reported in Manila in 1953-54, followed by the second outbreak that occurred after two years [1]. An epidemic occurred in Bangkok in 1958, although infrequent cases of DHF were reported in Thailand throughout 1950s [10].

In Southeast Asia, the first dengue epidemic reported in 1950s and unfortunately each passing epidemic had surpassed the previous one. Dengue has spread throughout the Southeast Asia over the last decade with eight countries within reported in 2003. In 2009, all the countries of Southeast Asia reported dengue except Korea. Eight South East Asian countries are now marked as hyperendemic regions where all four serotypes of Dengue are prevailing.

\section{Dengue in Bangladesh}

Dengue has recently become a major global health problem with a growing incidence in new countries and tropical areas. DF was first reported in Bangladesh in the mid-1960s and it continued till mid-1990s although no outbreak occurred during this period. The first dengue outbreak occurred in June 2000 during which clinics and hospitals began to be filled up by hemorrhagic fever patients. There were unprecedented 5551 cases reported, with 1.7\% case mortality rate in three divisional cities (Dhaka, Khulna and Chittagong) and 17 towns including 4385 (79.0\%) DF and 1166 (21.0\%) DHF cases. Dhaka and Chittagong showed low mortality rates and the ratio of DHF was low in Dhaka only [11]. In 2001, it slowed down and the reported number of deaths and cases reduced by $50 \%$. The number of cases increased by the following year 2002, during which 6132 cases were reported with $1.0 \%$ mortality rate [12]. Since then, the number of admitted patients in the hospitals exceeded three thousand mark up to five times till now, 6232 in 2002, 3934 in 2004, 3162 in 2015, 6060 in 2016, 10148 in 2018, and 100107 as of Nov 30, 2019 [13].

\section{Dengue Virus Classification}

The dengue virus belongs to the genus Flavivirus and the family Flaviviridae. This genus also contains several other viruses which are transmitted by mosquitoes and cause diseases to human. Yellow fever virus, West Nile virus, Japanese encephalitis virus and tick-borne encephalitis virus are some of the prominent virus belongs to the genus Flavivirus. The dengue virus contains positive polar single-stranded RNA and spreads with the help of Aedes (Stegomyia) spp., primarily Ae. aegypti and Ae. albopictus which are utilized as vectors for domiciliary and peridomestic transmission. On the other hand, arboreal Aedes mosquitoes are used as vectors for enzootic transmission and they are robustly confined in their host vertebrate range which includes primates only. Dengue virus is mainly of 4 serotypes including DEN-1, DEN-2, DEN-3, and DEN4 which are antigenically different although they share approximately $65 \%$ of their genomes. Recently, another serotype has emerged known as DEN-5. Although being antigenically different, all serotypes cause dengue with same clinical symptoms.

The DEN serotypes severely became hyperendemic after 
World War II. Explosive human population growth played an instrumental role in this rapid expansion of diversity along with unplanned urbanization, and huge human movement. Homologous recombination along with clonal expansion has contributed to the galloping genetic diversity. Co-infection of genetically distant Dengue virus strains occurs when different serotypes co-circulate reiteratively in one region. Another fact is that as Aedes aegypti gluttonize on various hosts, it makes the co-infection even more viable in both human host and mosquito. Recurrent human infection by different dengue virus serotypes is reported to be a common phenomenon [14]. It is surprising that putative recombinants were found within a single mosquito [15]. It also has been found that all serotypes can go through intra-serotype recombination no matter what the geographical region is although recombination does not confer any significant advantage [16].

During rudimentary period, only RNA fingerprinting was used to identify DEN strains with common spatial distribution [17]. However, the arrival of direct viral RNA sequencing not only accelerated the process of identification with greater proficiency but also with precise characterization. This advancement in technology helped in genotyping the virus strains with derivation of evolutionary relationship [18]. As the technology advanced, complete genome sequences were obtained with the details of precise phylogeny and additional topotypes. Among all the serotypes, DEN-3 is found the most fatal and exert severe complications. DEN-2 belonging to Southeast (SE) Asian genotype has the potential to cause dengue hemorrhagic fever (DHF) [19].

Among the four dengue serotypes, DEN1 and DEN2 are the prevalent ones found in the circulation of three metropolitan cities in Bangladesh [20]. As all the serotypes are predominant and persistent in the neighboring countries, there remains a high risk of secondary infection. DEN-2 strain which are responsible for most of the epidemics are classified into three distinct genotypes known as the American, SE Asian, and Indian Subcontinent genotypes [19].

\section{Clinical Manifestation of Dengue}

In the early stage of dengue virus infection, mild and undifferentiated "flu-like" fever symptoms can be observed just like the other viruses of Flaviviridae family. This fever is accompanied with symptoms similar to other diseases such as influenza, measles, Zika, chikungunya, yellow fever, and malaria which are caused by Flaviviridae family virus. About $20 \%$ of all infections are symptomatic and the symptoms covers a broad spectrum of mild to severe clinical manifestations [21].

Dengue virus infection can cause a wide spectrum of disorders in humans varying from unnoticeable or mild febrile illness to fatal and robust hemorrhagic fever. Clinical manifestation of the disease is very much irrespective of dengue virus serotypes although it can vary depending on various factors. In Dengue endemic regions, clinical manifestations are not always specific. Some of the risk factors that influence the number of patients having the disease during epidemic transmission include immune status, genetic background and age of the human host. One of the major problems in dengue diagnosis is that it can be asymptomatic in many cases. Dengue has three prominent clinical manifestations. According to WHO guideline of 1997, Dengue is classified into three types. They are:

1) Undifferentiated febrile illness (UF) or viral syndrome

2) Dengue fever (DF)

3) Dengue hemorrhagic fever (DHF)

a. DHF without shock

b. Dengue shock syndrome (DSS)

DF is considered as fever with symptoms like headache, myalgia, retro-orbital pain, rash, arthralgia and haemorrhagic manifestations. Differences between DHF and DF are based on thrombocytopenia, haemorrhagic manifestations, and plasma leakage. In case of DSS, symptoms must be same as DHF along with circulatory failure.

\section{Dengue Fever}

WHO defined dengue fever as an acute and sudden onset of febrile illness that persists for 2-7 days. The augmentation of temperature occurs initially and reach up to $102-105 \mathrm{~F}$ with mild bradycardia. It starts with a sudden onset of fever while having a mild sore throat. During this period, two or more symptoms like headache, retro-orbital pain, myalgia/arthralgia, maculopapular rash and petechiae are observed. Some nonspecific signs and symptoms include frontal headache, retro-orbital pain, body aches, nausea and vomiting, joint pains, weakness and rash (Table 1).

Anorexia and altered taste sensation, constipation are also occasionally reported and lymphadenopathy is common. It can also appear at the initial stage or just before the commencement of fever and disappear 1 to 2 days after the onset of symptoms. A second rash may be observed between 2 to 6 days of illness. Respiratory symptoms and Diarrhea occur infrequently which may be accompanied by concurrent infections. Intense itching and desquamation on the soles of the feet and the palms of the hands may occur. A DF patient faces thrombocytopenia which is later followed by lymphocytosis.

Infants and young children especially those who are less than 15 years of age may develop an undifferentiated febrile disease with concomitant maculopapular rash. Flushing is one of the common feature of this disease which is commonly 
observed on the neck, face and chest. Most dengue infections are mild and not apparent in young children. It is also similar to other common causes of febrile illnesses. Although DF is a very weakening disease, its prognosis is very much favorable. Liver enzyme levels are elevated mildly and both alanine aminotransferase and aspartate aminotransferase levels reach up to 500 to $1,000 \mathrm{U} /$ liter [22]. Tourniquet test shows a positive result World Health [23] during DF infection.

\section{Dengue Hemorrhagic Fever (DHF)}

DHF normally occur due to secondary dengue infection although in infants, it may occur due to primary infection. The patients have remarkably high viral loads as they have sluggish rate of virus containing immune complexes and viral load clearance compared to DF [24]. Initiation of DHF usually starts with a surprising elevation in temperature. Typical DHF cases are featured by four prominent clinical manifestations including high fever, haemorrhagic phenomena, and often hepatomegaly and circulatory failure. Hepatomegaly and splenomegaly are observed off and on, especially in infants. DHF has distinctive clinical laboratory symptoms including haemoconcentration and moderate to marked thrombocytopenia. The major pathophysiological change that separates DHF from DF is the plasma leakage. It is marked by elevated level of hematocrit or a serous effusion or hypoproteinemia. Between the days 3 and 8 of illness, platelet count drops down to $100,000 / \mathrm{mm} 3$. Plasma leakage also dictates the austerity of DHF disease (Table 1 ).

There are some persistent haematological alterations observed in DHF. They include complications like bone marrow suppression which results in thrombocytopenia and leukopenia. Thrombocytopathy consisting of thrombocytopenia and platelet dysfunction is caused by bone marrow suppression, immune injury, and infection of platelets by the dengue virus. Hemostatic changes in both DHF and DSS involve three major factors: vascular changes, thrombocytopenia, and coagulation disorders World Health [23] vascular fragility and thrombocytopenia are common phenomena in almost all the DHF patients. Another complication that persists with this condition is prolonged partial thromboplastin time. Decreased fibrinogen level, and increased levels of fibrinogen degradation products result in increased plasma leakage. Those who die of DHF tend to have GI hemorrhage. DHF patients normally have a short and uneventful convalescence. During this stage, many patients experience severe itching on their palms and soles.

\section{Dengue Shock Syndrome}

It is a severe form of dengue which has a mortality rate higher than the Dengue fever and Dengue hemorrhagic fever. DSS has symptoms similar to DHF. The condition of the patients who develop shock syndrome gets worse after 2-7 days. The onset of shock is acute and it starts with the fall of temperature. Apart from DHF, DSS also has symptoms like lower pulse pressure, long capillary refill, rapid and irregular pulse resulting in circulatory failure (Table 1). The skin becomes cold, congested and blotchy. Patients become lethargic initially and then become restless and either dies within 24 hours from the onset of shock or recover quickly after getting appropriate fluid replacement therapy. Recuperation from shock is uneventful and short although pleural effusions and ascites might last longer. Uncorrected shock can result in a convoluted course with concomitant metabolic acidosis. Severe bleeding can also take place from the GI tract and intracranial and other important organs, and as a consequence poor prognosis is observed. Metabolic and electrolyte disturbances may appear along with occasional encephalopathy.

\begin{tabular}{|c|c|c|}
\hline Disease name & Symptoms & Reference \\
\hline \multirow{8}{*}{ Dengue fever } & $>$ Headache & [25] \\
\hline & $>$ Myalgia & [8] \\
\hline & $>$ Rash & \\
\hline & $>$ Retro-orbital pain & \\
\hline & $\begin{array}{l}>\text { Platelet count } \\
\leq 150000 \text { cell } / \mathrm{mm}^{3}\end{array}$ & \\
\hline & $\begin{array}{l}>\text { Leukopenia }(\mathrm{WBC} \leq \\
\left.5,000 \text { cells } / \mathrm{mm}^{3}\right)\end{array}$ & \\
\hline & $\begin{array}{l}>\text { Hematocrit }(\mathrm{HCT}) \\
\text { rising } 5-10 \%\end{array}$ & \\
\hline & $>$ Petechiae & \\
\hline \multirow{7}{*}{$\begin{array}{c}\text { Dengue } \\
\text { hemorrhagic } \\
\text { fever }\end{array}$} & $>$ Plasma leakage & [26] \\
\hline & $\begin{array}{l}>\text { Thrombocytopenia } \\
\left(<100000 \text { cells } / \mathrm{mm}^{3}\right)\end{array}$ & [8] \\
\hline & $>\mathrm{HCT} \geq 20 \%$ & \\
\hline & $>$ Hepatomegaly & \\
\hline & $>$ Splenomegaly & \\
\hline & $>$ GI hemorrhage & \\
\hline & $>$ Vascular fragility & \\
\hline \multirow{4}{*}{$\begin{array}{l}\text { Dengue shock } \\
\text { syndrome }\end{array}$} & $>$ Lower pulse pressure & [27] \\
\hline & $>$ Cold, clammy skin & {$[8]$} \\
\hline & $>$ Restlessness & \\
\hline & $>$ Long capillary refill & \\
\hline
\end{tabular}

Table 1: Clinical symptoms of Dengue.

\section{Pathogenesis of Dengue}

The average incubation period of dengue virus is 4 to 7 days. During this period, the patient can be both symptomatic 
and asymptomatic. Expression of symptoms depends on several variables of which age, immunity status, virus strains are notable. This is followed by sudden onset of fever which is associated with viremia.

After the viremic phase, two types of fate can happen to a patient. He can either recover from fever or enter into leakage phase. Prolonged leakage phase leads to dengue hemorrhagic fever or dengue shock syndrome. Two premier pathophysiological changes are observed during DHF and DSS. One of them is vascular leakage which results in haemoconcentration and low pulse pressure. During secondary infection, cytokine Interleukin-2 and Tumor Necrosis Factor-alpha are released which enhances vascular permeability [28]. The second change is the loss of hemostasis due to vascular changes, thrombocytopenia and coagulopathy. The extremity of dengue infection is commensurate with the amount of circulating NS1 protein.

The dengue virus has the ability to replicate within mononuclear phagocytic cells like macrophage, monocyte, B cell. In addition, Mast cell, endothelial cell and dendritic cell are also get infected. The virus can infect peripheral blood leukocytes and organs like liver, spleen, lymph node, bone marrow, thymus, heart, kidney, stomach and lung. It can cross the blood brain barrier as well [29]. The more the infected cell, the more is the production of cytokines. TNF- $\alpha$ and IFN- $\alpha$ are the major cytokines that are released and these cytokines lead to complication like plasma leakage, increased vascular permeability, shock, hypovolemia and hemostatic abnormalities. These cytokines also activate other infected and non-infected dendritic cells.

Condition becomes Fatal when someone gets secondary infection by a different serotype. $2-4 \%$ individuals are suffered by some serious complications. A proposed mechanism is that the antibodies produced during the first infection cannot neutralize the different serotype of virus that enter during the second infection. In fact, the pre-existing antibodies help to exert even more severe infection via antibody dependent enhancement. Residual antibodies that are generated during the primary infection cross react with virus serotypes involved in secondary infection. They bind and coat the virus particles. Such coated virus particles are taken up more rapidly and efficiently by tissues, macrophage, dendritic cells and monocyte than the uncoated virions. This increased viral load results in enhanced antigen presentation to the T cell by the infected cell. As a consequence, Massive CD4+ and CD8+ cytotoxic lymphocytes activation and proliferation occurs which produce stunned $\mathrm{T}$ cell although $\mathrm{r}$ IFN- $\gamma$ expression remains low [30]. Lysis of infected monocytes is accountable for plasma leakage and hemorrhage that are mediated by cytotoxic lymphocytes. During second infection, C3 activator activates complement. By activating $\mathrm{C} 1, \mathrm{C} 2, \mathrm{C} 4$ cascades, reduced level of $\mathrm{C} 3$ is obtained. $\mathrm{C} 3 \mathrm{a}$ and $\mathrm{C} 5 \mathrm{a}$ generated from these cascade enhances vascular permeability [31].

\section{Clinical Diagnosis}

Clinical diagnosis of dengue is a very challenging task as there are several diseases whose symptoms overlap with dengue's symptom. Variation of symptom arises due to geographic region as well. There can be other disease-causing pathogen that can mimic the Dengue symptoms depending on what stage the patient is. Dengue virus infection can be asymptomatic as well.

\section{RT-PCR}

Molecular methods have provided more efficiency in terms of identifying DEN. Techniques like RT-PCR and nucleic acid hybridization are widely used with great success in diagnosing DEN infection. Scientists have been trying to develop several reverse transcriptase-polymerase chain reaction (RT-PCR) using different primers since 1990s. RT-PCR offers better sensitivity as well as provide results relatively quickly and using only one sample compared to virus isolation method. In situ RT-PCR provides the opportunity to detect dengue RNA even if it is from tissues that are embedded on paraffin.

Every nucleic acid detection assays are composed of three basic steps. They are extraction and purification, amplification and detection. As nested RT-PCR assay provides high sensitivities (80-100\%) and specificities (99-100\%), scientists opt for this technique. In this process, universal dengue primers against the $\mathrm{C} / \mathrm{prM}$ region of the genome are used for an initial reverse transcription and amplification step. It is followed by a nested PCR amplification loop that is serotype-specific [32]. Then one step multiplex RT-PCR came where a primer combination of four serotype specific oligonucleotide is used. It is a very useful alternative of nested PCR. Agarose gel electrophoresis is used to separate the products. One of the biggest advantage of reverse transcription-PCR amplification assay is that it is capable of detecting dengue RNA molecules within a mixture of other RNA molecules even if dengue virus is present in a very subtle amount. Biological amplification by culturing is a very slow, time-consuming process. On the other hand, RT-PCR can amplify millions of copies by enzymatic process within couple of hours. Moreover, during specimen preparation step, nucleic acid can be separated from proteins. Thus, reverse transcription-PCR amplification enables us to detect dengue virus during convalescence when serology dependent technique fail to detect DENV as circulating antibodies preclude its detection. The sensitivity of reverse transcription-PCR ranges between 80\%-100\% (Table-2). 


\begin{tabular}{|c|c|c|c|c|c|c|}
\hline $\begin{array}{c}\text { Type of } \\
\text { test }\end{array}$ & Test name & specimen & $\begin{array}{c}\text { Ability to } \\
\text { distinguish } \\
\text { primary and } \\
\text { secondary } \\
\text { infection }\end{array}$ & $\begin{array}{c}\text { Applicable } \\
\text { period }\end{array}$ & $\begin{array}{c}\text { Serotype } \\
\text { determination }\end{array}$ & References \\
\hline $\begin{array}{c}\text { Serological } \\
\text { test }\end{array}$ & MAC-ELISA & Serum, CSF & Yes & $>4-5$ Days & No & $\begin{array}{c}\text { Center for Disease } \\
\text { control and prevention, } \\
\text { USA } \\
{[17,38]}\end{array}$ \\
\hline & PRNT & Serum, CSF & Yes & $>4-5$ Days & Yes & {$[30,39]$} \\
\hline & HI test & Serum & Yes & $<5$ Days & No & {$[37,39]$} \\
\hline Ag test & Serum & No & $<3-4$ Days & & [37,39] \\
\hline Imminochro- & Serum & Yes & $<3-5$ Days & No & {$[37]$} \\
\hline Test & RT-PCR & $\begin{array}{c}\text { Serum or } \\
\text { plasma }\end{array}$ & No & $1-7$ Days & No & $\begin{array}{c}\text { Center for Disease } \\
\text { control and prevention, } \\
\text { USA }\end{array}$ \\
\hline & Multiplex PCR & $\begin{array}{c}\text { Serum or } \\
\text { plasma }\end{array}$ & No & Days & Yes & $\begin{array}{c}\text { Center for Disease } \\
\text { control and prevention, } \\
\text { USA }\end{array}$ \\
\hline
\end{tabular}

Table 2: Clinical diagnosis of dengue

\section{Real-time RT-PCR}

Real-time RT-PCR technique has gone through a lot of development over the years and now has reached a certain state. It is a one-step assay of quantifying viral RNA load that is very helpful in determining the pathogenesis of dengue disease [33]. It is preferable to RT-PCR since it takes less amount of time as well as less prone to contamination. In this method, the detection of reaction products are done by the use of fluorescence probes in lieu of gel electrophoresis. As sequence specific hybridization of the probe occur in Taqman real time PCR, it is very highly specific and depends on the homology with the targeted viral strain. Real time RT-PCR can be either singleplex or multiplex. The multiplex assay has the advantage of identifying all four serotypes with single reaction without any introduction of contamination.

\section{Haematological Tests}

A breakdown of platelet number and hematocrit level is a useful marker of acute phase dengue infection. It is a trademark feature of dengue hemorrhagic fever. During dengue fever platelet count drops below 100000 per $\mu \mathrm{L}$ between 3-8 days, just after the onset of illness thrombocytopenia is observed. An accretion in hematocrit level by $20 \%$ or more than the convalescent level indicates the presence of hypovolemia. It results from plasma leakage and enhanced vascular permeability.

\section{Serological tests}

\section{MAC-ELISA}

MAC-ELISA, a type of sandwich immunoassay, identifies and captures anti-dengue IgM antibodies. In this method, a microplate is coated with anti-human IgM antibodies which can recognize $\mu$ chain of immunoglobulin $M$. When the suspected patient's serum is applied to the plate, the serum IgM binds to the anti IgM antibody.

After incubation with dengue virus serotype as antigen, enzyme conjugated monoclonal or polyclonal dengue antibody that can convert a non-colored substrate into a colored compound is applied. When the substrate is incubated with the complex it generates a signal [34]. Both positive and negative control is used to set up a cut off value.

It is an ideal diagnostic method if the serum sample is collected in acute or convalescent period. The sensitivity is almost up to $98 \%$ and specificity is reported $90 \%$ on average 
[35]. However, it cannot separate the serotypes. Another major drawback of this technique is that as MAC-ELISA identifies antibody and it takes couple of days since the infection to produce antibody, it can produce false negative result if it is done just after the onset of infection (Table 2 ). Although cross reactivity with other flavivirus does not pose any problem, serum sample of patients having malaria or dengue infection previously can give false positive result [36]. Standardization of reagents is mostly responsible for false result.

\section{IgM/IgG ratio}

IgM antibody is the first antibody to be produced within 4-5 days of fever onset. It is followed by an upsurge of IgG antibody within 7-10 days of fever onset. The amount of IgM remains higher than IgG during primary infection. ELISA is the most common technique used to measure IgM and IgG antibodies. If the IgM/IgG OD ratio becomes greater than 1.2; the infection considered as primary infection.

IgG acts as memory antibody when dengue infection occurs second time. During secondary infection, IgG antibody recognizes the viral particles and triggers its level whereas IgM antibody is produced in minimal level and sustains for short period of time. IgG level remains higher than IgM during secondary infection. If the $\operatorname{IgM} / \operatorname{IgG}$ ratio is lower than 1.2 or 1.4 then it is considered as secondary infection [7].

\section{Detection of NS1}

The nonstructural protein 1 (NS1) a conserved glycoprotein is secreted as hexamer from the cells infected by DENV. It is found in 2 forms: either anchored to the membrane (mNS1) or freed into the extracellular medium in its soluble form (sNS1) [36].

ELISA antigen capture technique is used to identify and quantify NS1. For this, the ELISA plate is coated with anti NS1 antibody which will recognize the NS1 protein of the virus sample. It is then followed by the addition of a secondary antibody which is coupled to an enzyme that can convert a non-colored substrate to a colored compound.

The NS1 glycoprotein is generated by all the flavivirus. Since IgM, IgG takes a few days to be generated whereas NS1 is present in acute viraemic phase of infection, NS1 detection becomes highly useful for early diagnosis [40]. Serum samples taken within 1-3 days after the infection will show IgM negative result which is misleading but will show NS1 positive result. Characteristics including early presence, specificity to dengue and abundance in sera make it a perfect candidate for rapid diagnostic test [41]. However, NS1 cannot be detected if the patient's serum is taken 5-7 days after infection and it cannot differentiate different serotypes. The detection of NS1 in secondary infection becomes very difficult due to small window as NS1 forms complex with the pre-existing IgG antibody [42]. Another major issue is its' sensitivity which varies with serotype meaning that the newly arrived serotypes may not be detected [43]. Therefore, NS1 detection test is advantageous for early detection of dengue although its sensitivity is yet to be determined.

\section{Conclusion}

There are different ways to diagnose dengue. Although many methods are available, they differ in accuracy, sensitivity and specificity. It's a major problem in identifying and measuring the severity of the disease. Sometimes patients are asymptomatic and so they remain undiagnosed. Regions where dengue is not endemic or predominant dengue symptoms are clinically mistaken as other disease. This is one of the main reasons behind low number of reported case in Africa [44-51]. Dengue patients are misdiagnosed as a febrile disease when they don't have any typical symptoms or they catch dengue outside the dengue season. Combination of extensive amount of diagnosis and improvement of accuracy are needed for improving surveillance on dengue. Extensive diagnosis helps to identify pandemic or prominent region of dengue. It also helps to the policy makers to make policy interventions and take necessary steps so that it cannot spread and rapid diagnosis can be made available [52-54].

\section{References}

1. Halstead SB (1980) Dengue haemorrhagic fever--a public health problem and a field for research. Bull World Health Organ 58(1): 1-21.

2. Guha-Sapir D, Schimmer B (2005) Dengue fever: new paradigms for a changing epidemiology. Emerg Themes Epidemiol 2: 1.

3. Hsieh YH, Cheng YS (2006) Real-time forecast of multiphase outbreak. Emerg Infect Dis 12(1): 122-127.

4. Simmons CP, Farrar JJ, Nguyen VV, Wills B (2012) Dengue. N Engl J Med 366: 1423-1432.

5. Gubler DJ (1998) Dengue and dengue hemorrhagic fever. Clin Microbiol Rev 11(3): 480-496.

6. Sabin AB (1952) Research on dengue during World War II. Am J Trop Med Hyg 1(1): 30-50.

7. Falconar AK, De Plata E, Romero-Vivas CM (2006) Altered enzyme-linked immunosorbent assay immunoglobulin M (IgM)/IgG optical density ratios can correctly classify all primary or secondary dengue virus infections 1 day after the onset of symptoms, when all of the viruses can 
be isolated. Clin Vaccine Immunol 13(9): 1044-1051.

8. Organization WH (2011) Comprehensive guideline for prevention and control of dengue and dengue haemorrhagic fever.

9. Karamchandani PV (1946) Dengue group of fevers in India. Lancet 1(6386): 92.

10. Hammon WM (1973) Dengue hemorrhagic fever--do we know its cause? Am J Trop Med Hyg 22(1): 82-91.

11. Yunus EB, Bangali AM, Mahmood MAH, Rahman MM, Chowdhury AR, et al. (2001) Dengue Outbreak 2000 in Bangladesh: From Speculation to Reality and Exercises. 25: $15-20$.

12. Sharmin S, Viennet E, Glass K, Harley D (2015) The emergence of dengue in Bangladesh: epidemiology, challenges and future disease risk. Trans R Soc Trop Med Hyg 109(10): 619-627.

13. Shirin T, Muraduzzaman AKM, Alam AN, Sultana S, Siddiqua M, et al. (2019) Largest dengue outbreak of the decade with high fatality may be due to reemergence of DEN-3 serotype in Dhaka, Bangladesh, necessitating immediate public health attention. New Microbes New Infect 29: 100511.

14. Lorono-Pino MA, Cropp CB, Farfan JA, Vorndam AV, Rodriguez-Angulo EM, et al. (1999) Common occurrence of concurrent infections by multiple dengue virus serotypes. Am J Trop Med Hyg 61(5): 725-30.

15. Craig S, Thu HM, Lowry K, Wang XF, Holmes EC, et al. (2003) Diverse dengue type 2 virus populations contain recombinant and both parental viruses in a single mosquito host. Journal of virology 77(7): 4463-4467.

16. Worobey M, Rambaut A, Holmes EC (1999) Widespread intra-serotype recombination in natural populations of dengue virus. Proc Natl Acad Sci U S A 96(13): 73527357.

17. Vezza AC, Rosen L, Repik P, Dalrymple J, Bishop DH (1980) Characterization of the viral RNA species of prototype dengue viruses. Am J Trop Med Hyg 29(4): 643-652.

18. Rico-Hesse $\mathrm{R}$ (1990) Molecular evolution and distribution of dengue viruses type 1 and 2 in nature. Virology 174(2): 479-493.

19. Armstrong PM, Rico-Hesse R (2003) Efficiency of dengue serotype 2 virus strains to infect and disseminate in Aedes aegypti. Am J Trop Med Hyg 68(5): 539-544.

20. Muraduzzaman AKM, Alam AN, Sultana S, Siddiqua M, Khan MH, etal. (2018) Circulating dengue virus serotypes in Bangladesh from 2013 to 2016. Virusdisease 29(3): 303-307.

21. Yacoub S, Lam PK, Le V, Le TL, Ha NT, et al. (2016) Association of Microvascular Function and Endothelial Biomarkers With Clinical Outcome in Dengue: An Observational Study. J Infect Dis 214(5): 697-706.

22. Dietz V, Gubler DJ, Ortiz S, Kuno G, Casta-Velez A, et al. (1996) The 1986 dengue and dengue hemorrhagic fever epidemic in Puerto Rico: epidemiologic and clinical observations. P R Health Sci J 15(3): 201-210.

23. World Health $O$ (1997) Dengue haemorrhagic fever: diagnosis, treatment, prevention and control. 2nd ed ed. Geneva: World Health Organization.

24. Wang WK, Chen HL, Yang CF, Hsieh SC, Juan CC, et al. (2006) Slower rates of clearance of viral load and viruscontaining immune complexes in patients with dengue hemorrhagic fever. Clin Infect Dis 43(8): 1023-1030.

25. Kalayanarooj S (2011) Clinical Manifestations and Management of Dengue/DHF/DSS. Trop Med Health 39(4): 83-87.

26. Tsai TT, Chuang YJ, Lin YS, Wan SW, Chen CL, et al. (2013) An emerging role for the anti-inflammatory cytokine interleukin-10 in dengue virus infection. J Biomed Sci 20(1): 40 .

27. Ngo NT, Cao XT, Kneen R, Wills B, Nguyen VM, et al. (2001) Acute management of dengue shock syndrome: a randomized double-blind comparison of 4 intravenous fluid regimens in the first hour. Clin Infect Dis 32(2): 204-213.

28. Chakravarti A, Kumaria R (2006) Circulating levels of tumour necrosis factor-alpha \& interferon-gamma in patients with dengue \& dengue haemorrhagic fever during an outbreak. Indian J Med Res 123(1): 25-30.

29. Hayes EB, Gubler DJ (1992) Dengue and dengue hemorrhagic fever. Pediatr Infect Dis J 11(3): 311-317.

30. Halstead SB (1988) Pathogenesis of dengue: challenges to molecular biology. Science 239(4839): 476-81.

31. Bokisch VA, Top FH, Russell PK, Dixon FJ, MullerEberhard HJ (1973) The potential pathogenic role of complement in dengue hemorrhagic shock syndrome. N Engl J Med 289(19): 996-1000.

32. Lanciotti RS, Calisher CH, Gubler DJ, Chang GJ, Vorndam AV (1992) Rapid detection and typing of dengue viruses from clinical samples by using reverse transcriptasepolymerase chain reaction. J Clin Microbiol 30: 545-551.

33. Vaughn DW, Green S, Kalayanarooj S, Innis BL, 
Nimmannitya S, et al. (2000) Dengue viremia titer, antibody response pattern, and virus serotype correlate with disease severity. J Infect Dis 181(1): 2-9.

34. Nawa M, Takasaki T, Yamada KI, Akatsuka T, Kurane I (2001) Development of dengue IgM-capture enzymelinked immunosorbent assay with higher sensitivity using monoclonal detection antibody. J Virol Methods 92(1): 65-70.

35. Hunsperger EA, Yoksan S, Buchy P, Nguyen VC, Sekaran SD, et al. (2009) Evaluation of commercially available anti-dengue virus immunoglobulin M tests. Emerg Infect Dis 15(3): 436-440.

36. Young PR, Hilditch PA, Bletchly C, Halloran W (2000) An antigen capture enzyme-linked immunosorbent assay reveals high levels of the dengue virus protein NS1 in the sera of infected patients. J Clin Microbiol 38(3): 10531057.

37. Chanama S, Anantapreecha S, A-nuegoonpipat A, Sagnasang A, Kurane I, Sawanpanyalert P (2004) Analysis of specific IgM responses in secondary dengue virus infections: levels and positive rates in comparison with primary infections. J Clin Virol 31(3): 185-189.

38. WHO (2009) Dengue guidelines for diagnosis, treatment, prevention and control: new edition. Geneva: World Health Organization.

39. Innis BL, Nisalak A, Nimmannitya S, Kusalerdchariya S, Chongswasdi V, et al. (1989) An enzyme-linked immunosorbent assay to characterize dengue infections where dengue and Japanese encephalitis co-circulate. Am J Trop Med Hyg 40(4):418-27.

40. Janeczko D, Czyzyk A, Kopczynski J, Krzyzanowski M (1991) Risk factors of cardiovascular death in diabetic patients. Diabet Med 8(52): 100-103.

41. Raafat N, Blacksell SD, Maude RJ (2019) A review of dengue diagnostics and implications for surveillance and control. Trans R Soc Trop Med Hyg 113(11): 653660.

42. Da Costa VG, Marques-Silva AC, Moreli ML (2014) A metaanalysis of the diagnostic accuracy of two commercial NS1 antigen ELISA tests for early dengue virus detection. PLoS One 9(4): e94655.

43. Hunsperger EA, Sharp TM, Lalita P, Tikomaidraubuta K, Cardoso YR, et al. (2016) Use of a Rapid Test for Diagnosis of Dengue during Suspected Dengue Outbreaks in Resource-Limited Regions. J Clin Microbiol 54(8): 20902095.

44. Bhatt S, Gething PW, Brady OJ, Messina JP, Farlow AW, et al. (2013) The global distribution and burden of dengue. Nature 496(7446): 504-507.

45. Aihara H, Takasaki T, Toyosaki-Maeda T, Suzuki R, Okuno Y, et al. (2000) T-cell activation and induction of antibodies and memory $\mathrm{T}$ cells by immunization with inactivated Japanese encephalitis vaccine. Viral Immunol 13(2): 179-186.

46. Beckett CG, Tjaden J, Burgess T, Danko JR, Tamminga C, et al. (2011) Evaluation of a prototype dengue-1 DNA vaccine in a Phase 1 clinical trial. Vaccine 29(5): 960868.

47. Burton DR, Williamson RA, Parren PW (2000) Antibody and virus: binding and neutralization. Virology 270(1): 1-3.

48. Durbin AP, Kirkpatrick BD, Pierce KK, Carmolli MP, Tibery CM, et al. (2016) A 12-Month-Interval Dosing Study in Adults Indicates That a Single Dose of the National Institute of Allergy and Infectious Diseases Tetravalent Dengue Vaccine Induces a Robust Neutralizing Antibody Response. J Infect Dis 214(6): 832-835.

49. Durbin AP, Kirkpatrick BD, Pierce KK, Elwood D, Larsson CJ, et al. (2013) A single dose of any of four different live attenuated tetravalent dengue vaccines is safe and immunogenic in flavivirus-naive adults: a randomized, double-blind clinical trial. J Infect Dis 207(6): 957-965.

50. Durbin AP, Whitehead SS (2011) Next-generation dengue vaccines: novel strategies currently under development. Viruses 3(10): 1800-1814.

51. Gubler DJ (2002) Epidemic dengue/dengue hemorrhagic fever as a public health, social and economic problem in the 21st century. Trends Microbiol 10(2): 100-103.

52. Gubler DJ, Clark GG (1995) Dengue/dengue hemorrhagic fever: the emergence of a global health problem. Emerg Infect Dis 1(2): 55-57.

53. Jechlinger $W$ (2006) Optimization and delivery of plasmid DNA for vaccination. Expert Rev Vaccines 5(6): 803-825.

54. Wilder-Smith A, Gubler DJ (2008) Geographic expansion of dengue: the impact of international travel. Med Clin North Am 92(6): 1377-1390. 